\title{
ASSOCIATION OF SERUM HOMOCYSTEINE IN DIABETIC NEUROPATHY
}

\section{RUJASWINI T ${ }^{1}$, PRAVEEN D², RANADHEER CHOWDARY ${ }^{2}$, VIJEY AANANDHI $\mathbf{M}^{3}$, SHANMUGASUNDARAM P4*}

${ }^{1}$ Department of Pharmacy Practice, School of Pharmaceutical Sciences, Vels Institute of Science Technology and Advanced Studies (VISTAS), Chennai, Tamil Nadu, India. ${ }^{2}$ Research Scholar, School of Pharmaceutical Sciences, Vels Institute of Science Technology and Advanced Studies (VISTAS), Chennai, Tamil Nadu, India. ${ }^{3}$ Department of Pharmaceutical Chemistry and Analysis, School of Pharmaceutical Sciences, Vels Institute of Science Technology and Advanced Studies (VISTAS), Chennai, Tamil Nadu, India. ${ }^{4}$ School of Pharmaceutical Sciences, Vels Institute of Science Technology and Advanced Studies (VISTAS), Chennai, Tamil Nadu, India. Email: samsimahe@gmail.com

Received: 10 October 2018, Revised and Accepted: 11 December 2018

\section{ABSTRACT}

Aims and Objectives: The main aim of the study was to find out the association of serum homocysteine (HCY) in diabetic neuropathy patients.

Methods: All the patients who were diagnosed with Type II diabetes mellitus will be included. Their serum levels of fasting blood sugar, postprandial blood sugar, glycated hemoglobin, and associated blood parameters will be assessed. Diabetic neuropathy will be confirmed using nerve conduction testing, electromyography, and quantitative sensory testing with clinically correlated. The serum HCY levels will be measured and correlated with other blood parameters.

Results: Of 1000 patients, 46 were Type I diabetic and 954 were Type II. The prevalence of neuropathy in diabetic patients was 156 . Mean serum HCY without diabetic neuropathy was $6.8+2.9$ and serum HCY with diabetic neuropathy was $21.6+0.29$ and p value was found to be 0.0017 . The correlation between serum HCY and diabetic neuropathy was found to be 14.5 with $\mathrm{p}=0.001$.

Conclusion: There has been a significant increase of HCY in diabetic patients. It can be clearly seen that elevated serum HCY level has led to some of the complications of diabetic neuropathy.

Keywords: Diabetes, Diabetic neuropathy, Homocysteine, Hyperhomocysteinemia, Methionine.

(C) 2018 The Authors. Published by Innovare Academic Sciences Pvt Ltd. This is an open access article under the CC BY license (http://creativecommons. org/licenses/by/4. 0/) DOI: http://dx.doi.org/10.22159/ajpcr.2018.v11s4.31741

\section{INTRODUCTION}

Diabetes is a disease in which blood glucose levels are too high. In the food, we take glucose was present. Insulin a hormone which acts as a mediator by helping the glucose gets into cells to give them energy. In Type 1 diabetic condition, insulin is of little amount or not produced by the pancreas, whereas Type 2 diabetes has a chronic condition affected the way the body processes blood sugar.

Type 2 diabetes mellitus (T2DM) is a chronic metabolic disorder [1]. High blood sugar, insulin resistance, and relative lack of insulin are a few characteristics of type 2 diabetic mellitus. Common symptoms are weight loss, frequent uncontrolled urination, and thirst.

Nerve dysfunction occurs in the legs and feet often in uncontrolled diabetic patients, which is known as diabetic neuropathy [2,3]. Nerve damage occurs in diabetes slowly without any symptoms. In the hands, arms, feet, and legs symptoms such as pain, tingling, or numbness occur in fewer. Roughly $50 \%$ of patients with diabetes suffer from diabetic neuropathy [4].

Homocysteine (HCY) is a common amino acid in blood. Methionine is converted to HCY and to cysteine, then finally to protein with the help of folic acid or Vitamin B6. Men above the age of 50 have increased in HCY levels when compared to women [5].

Structure of HCY [6]<smiles>NC(CCS)C(=O)O</smiles>

Chemical formula $-\mathrm{C}_{4} \mathrm{H}_{9} \mathrm{NO}_{2} \mathrm{~S}$

\section{Reference range}

HCY level may vary with the technique used so reference value can also be varied. Reference values by age are as follows [7]:

- For the people between 0 and 30 years of age Hcy level: 4.6-8.1 $\mu \mathrm{mol} / \mathrm{L}$

- For the people between 30 and 59 years of age Hcy level: 6.3-11.2 $\mu \mathrm{mol} / \mathrm{L}$ in males, whereas 4-5-7.9 $\mu \mathrm{mol} / \mathrm{L}$ in females.

- $\quad$ For the people $>59$ years of age Hcy level: 5.8-11.9 $\mu \mathrm{mol} / \mathrm{L}$.

HCY levels are checked through blood tests. A normal level of HCY in per liter of blood is approximately between 4.4 and $10.8 \mu \mathrm{mol} / \mathrm{L}$. Hyperhomocysteinemia is a condition where serum HCY level is above $15 \mu \mathrm{mol} / \mathrm{L}$ [8]. When any one of the Vitamin $\mathrm{B}_{12}$, Vitamin $\mathrm{B}_{6}$, and folic acid deficiency occurs, plasma HCY levels increase in the body [9].

Especially in the aged, elevation in HCY is common [10]. Endothelial cell injury may occur with high level of HCY in the blood, which leads to inflammation in the blood vessels, which, in turn, may lead to atherogenesis. The risk of stroke, peripheral vascular disease (PVD), and venous thromboembolism increases with hyperhomocysteinemia in the body [11]. Elevated levels of HCY have increased risk of cardiovascular disease (CVD) and arteriosclerosis [12]. High level of HCY is also responsible for CVD in adolescents [13]. High serum HCY level can develop vascular changes, especially in diabetes [14].

\section{Metabolism of homocysteine}

HCY metabolism is at the intersection of two metabolic pathways: Remethylation and transsulfuration [15] (Scheme 1).

HCY is biosynthesized from methionine by a multistep process. Remethylation is a process where HCY is converted to methionine in the presence of the enzyme methionine synthase and transsulfuration 


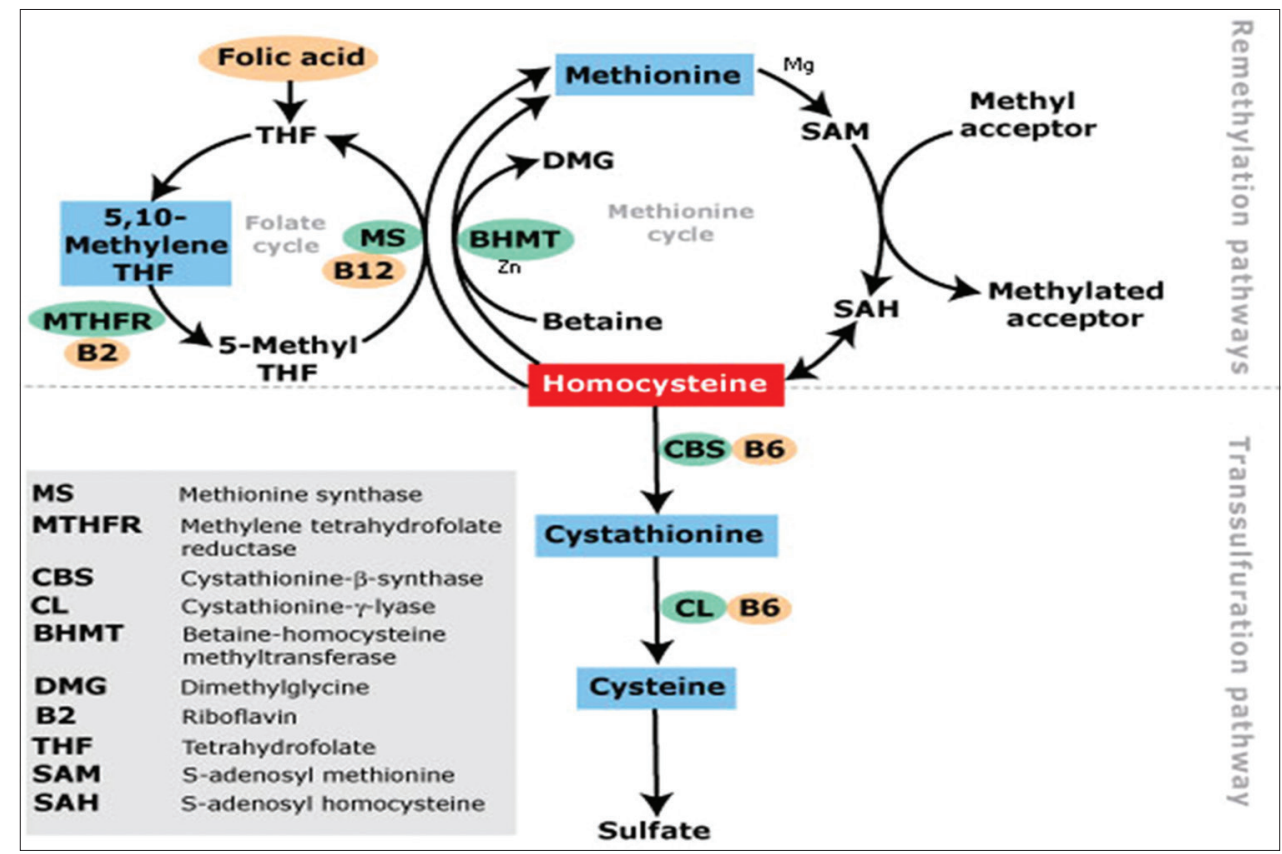

Scheme 1: Homocysteine metabolism

is a process where HCY is converted to cystathionine by the presence of enzyme cystathionine b-synthase. In the remethylation process of homocysteine, Vitamin B12 is required. HCY transsulfuration requires Vitamin B6 [16].

\section{Relation between HCY and diabetic neuropathy}

For the diabetic patient's serum levels of fasting blood sugar (FBS), postprandial blood sugar (PPBS), glycated hemoglobin (HbA1C), and associated blood parameters will be assessed [17]. Myocardial risk and heart attacks are noted in diabetic patients with dyslipidemia. HbA1C and lipid profile test to be monitored every 3 months for the diabetic patients to understand and prevent heart attacks [18]. Diabetic neuropathy will be confirmed using nerve conduction testing (NCT), electromyography (EMG), and quantitative sensory testing with clinically correlated. The serum HCY levels will be quantified and correlated with other blood parameters. Monitoring serum HCY concentration, as well as folate and Vitamin B12 status in T2DM patients, could be used as an indicator for assessing microvascular risk in DM [19]. Higher HCY levels are found in diabetics who have developed micro-/macro-vascular complications, and it is highly correlated with $\mathrm{HbA1c}$ and serum thyroglobulin which are also indicators of poor diabetic control [20]. For prediction of cardiovascular risk events in Type II diabetic patients, serum HCY can be independently used [21]. Prospective study in the large population would be suitable to elucidate the HCY function in the pathogenesis of diabetic neuropathy [22].

Macroangiopathy and nephropathy are mostly noted with elevated HCY levels in Type 2 diabetes [23]. HCY is clearly associated with the occurrence of diabetic neuropathy [24]. Many researchers ended in their study that HCY is more helpful in assessing CVD than cholesterol. HCY is a sensitive biomarker for both flat deficiency and CVD [25]. Many studies also concluded that the risk of atherosclerosis is higher with the increase in HCY level, irrespective of either cholesterol is normal or elevated.

Aim

This study aims to study the clinical correlation of serum HCY and diabetic neuropathy.

\section{Objectives}

The objectives of this study were as follows:

1. To evaluate the prevalence of diabetic neuropathy.

2. To evaluate the serum HCY levels in all diabetic patients.

\section{METHODS}

Study site

This study was conducted at the General Medicine Department of ESI Hospital, Ayanavaram.

\section{Sample size}

A total of 1000 diabetic patients will be assessed for symptoms of diabetic neuropathy. Among them, patient who is given a clear diagnosis based on clinical evaluation by the physician is involved in the study.

$\mathrm{S} . \mathrm{S}=\mathrm{Z}^{2} \mathrm{P}(1-\mathrm{P}) / \mathrm{C}^{2}$

$\mathrm{Z}=1.96, \mathrm{C} . \mathrm{I}=95 \%, \mathrm{P}=0.5$

$S . S=384$

New S.S = S.S $/ 1+($ S.S-1) $/$ Pop

S.S $=384$, Pop $=200$

New S.S $=132$

Study duration

The study duration was September 2017-April 2018

Study design

This was a prospective observational study.

\section{Methods involved}

All the patients who were diagnosed with T2DM will be included in the study. Their serum levels of FBS, PPBS, HbA1C, and associated blood parameters will be assessed. Diabetic neuropathy will be confirmed using NCT, EMG, and quantitative sensory testing with clinically correlated. The serum HCY levels will be measured and correlated with other blood parameters.

\section{Plan of the study}

The complete study was planned to be carried out for a period of 8 months from September 2017 to April 2018. 
Table 1: Baseline characteristics

\begin{tabular}{|c|c|c|c|c|}
\hline S. No. & Parameters & DM without neuropathy $(n=844)$ & DM with neuropathy $(n=156)$ & $\mathbf{p}$ \\
\hline 1. & Male & 584 & 50 & - \\
\hline 2. & Age (years) & $57.6 \pm 3.2$ & $51.6 \pm 2.91$ & 0.0527 \\
\hline 3. & BMI & $24.1 \pm 2.7$ & $23.2 \pm 1.8$ & 0.8217 \\
\hline 4. & Mean distribution of disease (years) & $3.18 \pm 1.6$ & $5.71 \pm 1.8$ & $0.0439 *$ \\
\hline 5. & Mean $\mathrm{HbA} 1 \mathrm{C} \%$ & $6.92 \pm 1.8$ & $8.14 \pm 1.2$ & $0.0327^{*}$ \\
\hline 6. & Mean serum homocysteine $(\mu \mathrm{mol} / \mathrm{l})$ & $6.8 \pm 2.9$ & $21.6 \pm 0.29$ & $0.0017^{*}$ \\
\hline
\end{tabular}

\section{Patient selection}

Inclusion criteria

The following criteria were included in the study:

- All diabetes patients were selected for the study.

- All patients who are $>18$ years of age.

- Willingness to give consent to the study.

\section{Exclusion criteria}

The following criteria were excluded from the study:

- Pregnant and nursing women.

- Patients on antituberculosis therapy.

- Patients who are diagnosed with peripheral neuropathy and PVD.

- Patients who are diagnosed with cardiac disorder, stroke, and Alzheimer's disease.

- $\quad$ Palliative patients are excluded.

An informed consent has been obtained from all the included patients. Patient confidentiality has been maintained throughout this study. The work has been approved by the Institutional Ethics Committee VISTASSPS/IEC/V/2017/06.

\section{Statistical analysis}

Correlation and regression analysis will be carried out with a confidence interval of $95 \%$ and $\mathrm{p}<0.05$ is considered statistically significant.

\section{RESULTS}

Fig. 1 shows that more male patients participated in a study than female, and among 1000 enrolled participants, 634 were male and 366 were female.

From Fig. 2, it can be noticed that there are more diabetic patients among the age group of 50-70 years.

Among 1000 enrolled patients, 46 were of Type I and the rest 954 were noticed with Type II diabetic patients. Fig. 3 shows that Type II diabetes was more prone than Type I diabetic patients.

Among 1000 enrolled diabetic patients, 156 were found to be with neuropathy and 844 without neuropathy. Fig. 4 shows that diabetic neuropathy patients are less prevalent when compared to the patients without neuropathy.

$\%$ Prevalence $=$ Number of patients affected $/$ population

$$
\begin{aligned}
& =156 / 1000 \\
& =15.6
\end{aligned}
$$

Table 1 shows the baseline characteristics that are analyzed in the diabetic patients with and without neuropathy.

Correlation of serum HCY between diabetic neuropathy and diabetic without neuropathy

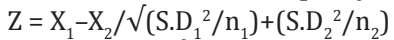

$\mathrm{Z}=21.6-6.8 / \sqrt{(2.9)^{2} / 844+(2.89)^{2} / 156}$

$Z=14.8 / \sqrt{ }(8.41 / 844)+(8.41 / 156)$

$\mathrm{Z}=14.8 / \sqrt{0.0997+0.054}$

$\mathrm{Z}=14.8 / 1.02=14.5$

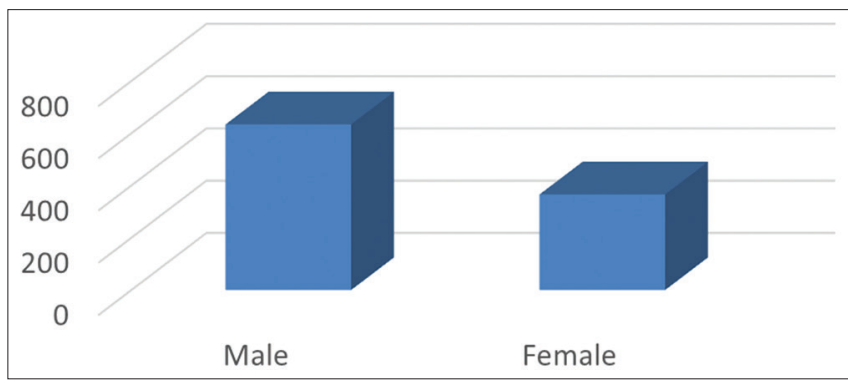

Fig. 1: Gender distribution

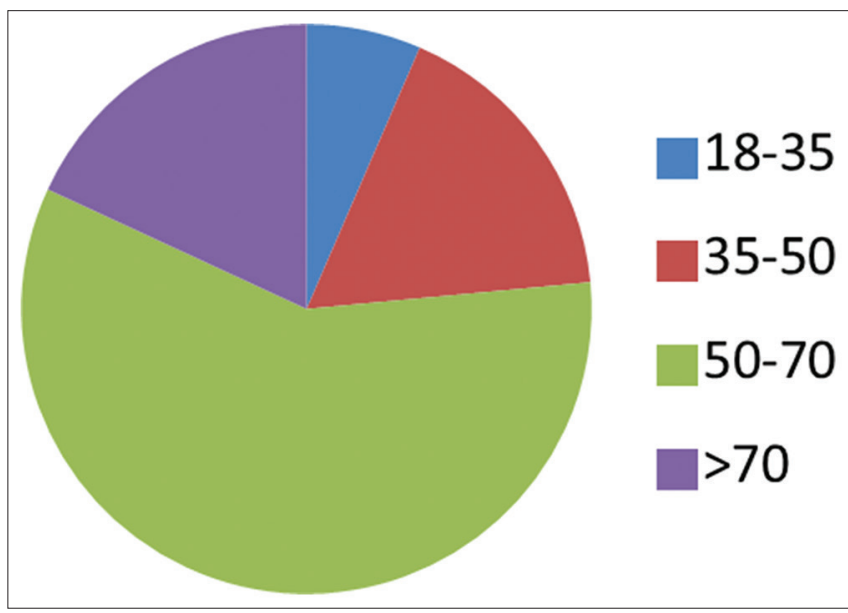

Fig. 2: Age distribution

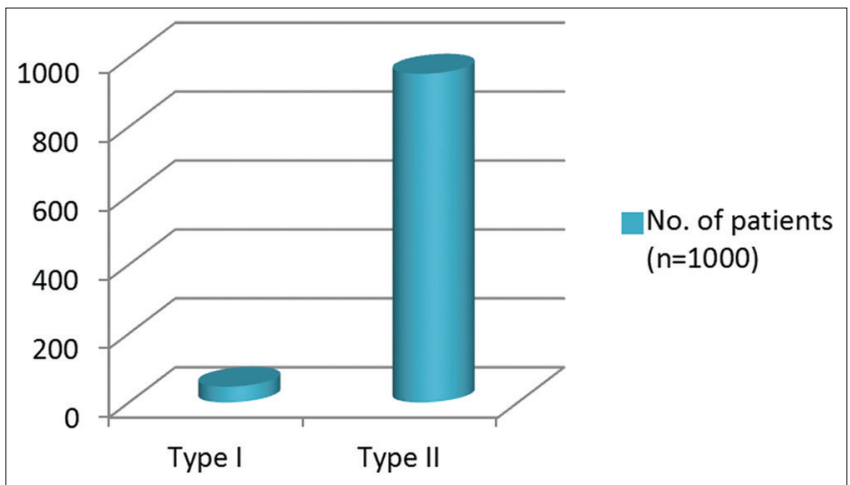

Fig. 3: Diabetes mellitus type

$\mathrm{Z}=14.5$

$p$ value was found to be 0.001 for $Z=14.5$

${ }^{*} \mathrm{p}<0.001$ was considered to be statistically significant

Table 2 shows that age, gender, body mass index (BMI), duration of disease, $\mathrm{HbA} 1 \mathrm{C} \%$, serum homocysteine, biguanide, and insulin use affecting the nerve conduction velocity (NCV). 
Table 2: Nerve conduction velocity

\begin{tabular}{llll}
\hline S. No. & Parameter & Normal NCV (n=31) & Abnormal NCV (n=125) \\
\hline 1. & Mean age (years) & $49.32 \pm 2.81$ & $52.18 \pm 3.21$ \\
2. & BMI & $22.7 \pm 2.8$ & $24.1 \pm 2.7$ \\
3. & Duration of disease (years) & $4.9 \pm 2.1$ & $5.91 \pm 1.8$ \\
4. & Mean HbA1C\% & $7.1 \pm 2.6$ & $9.8 \pm 1.8$ \\
5. & Mean serum homocysteine $(\mu \mathrm{mol} / \mathrm{l})$ & $13.2 \pm 0.87$ & $26.16 \pm 0.29$ \\
6. & Biguanide & 31 & $0.0497^{*}$ \\
7. & Using insulin & 07 & $0.0308^{*}$ \\
\hline
\end{tabular}

All values are mean \pm SEM. SEM: Standard error of the mean, NCV: Nerve conduction velocity

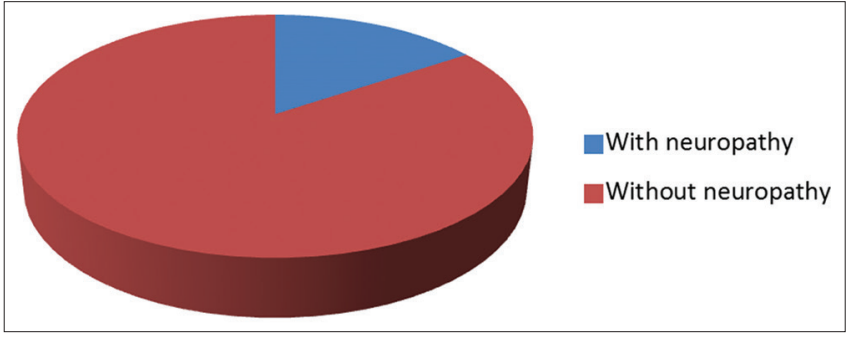

Fig. 4: Prevalence of diabetic neuropathy

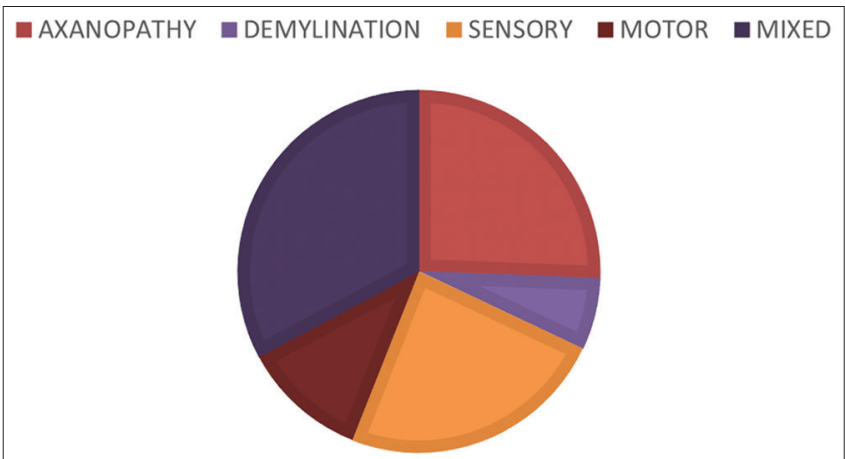

Fig. 5: Pattern of nerve conduction velocity abnormalities

Risk of abnormal NCV increased with age. It was clearly noted that the risk of NCV abnormalities in diabetes was high with increased HCY levels in the body.

${ }^{*} \mathrm{p}<0.001$ was considered to be statistically significant.

Correlation of Serum. HCY for NCV (normal and abnormal)

$\mathrm{Z}=26.6-13.2 / \sqrt{ }(0.87)^{2} / 31+(2.9)^{2} / 125$

$=13.4 / \sqrt{ } 0.0244+0.067$

$=13.4 / \sqrt{ } 1.58=8.48$

$\mathrm{Z}=8.48$

$\mathrm{p}$ value of $\mathrm{Z}=8.48$ was found to be 0.001 .

${ }^{*} \mathrm{p}<0.001$ was considered to be statistically significant.

Fig. 5 shows the pattern of NCV abnormalities.

In the current study, in the presence of a total of 156 diabetes with neuropathy, NCV was found to be abnormal in 125 patients and normal in 31 patients. Among those with abnormal NCV, 32 patients were found to have axonopathy, 8 with demyelination pattern, motor abnormalities diagnosed in 14, 30 had sensory abnormalities, and 41 had mixed abnormalities.

\section{DISCUSSION}

Diabetic neuropathy is seen in many patients who have uncontrolled diabetes. Nerve dysfunction due to high blood glucose levels, is common and complex nowadays in the diabetic patients. Abnormal levels of serum HCY involve the development of the diabetic neuropathy in Type 2 diabetic patients. Several studies showed possible association between HCY and diabetic neuropathy in patients with Type 2 diabetes. A study also reports that an HCY level progressively increased with the development of diabetic nephropathy [26].

Diabetic peripheral neuropathy may be asymptomatic in $50 \%$ of cases, which is one of the most important complications of diabetes. $\mathrm{HCY}$ level in diabetic patients is an important biomarker in assessing microvascular complications [27]. For diabetes, screening and treatment with folic acid to be preferred to control the abnormalities of HCY in the body.

Most symptoms are not appearing on the early stage of diabetic neuropathy. For a diagnosing diabetic neuropathy, careful examination of the lower limbs is required. Patients with peripheral neuropathy must be considered at risk of foot ulceration and must receive preventive education and podiatric care. There was a significant increase of HCY in diabetic patients and its positive significant correlations with BMI, HbA1c, glucose, triglyceride, urea, and creatinine [28].

\section{CONCLUSION}

Our study reveals that there was a definite relationship between elevated serum HCY and diabetic neuropathy. There has been a significant increase of HCY in diabetic patients and its positive significant correlations with BMI, HbA1c, and duration of disease. It can be clearly seen that elevated serum HCY level has led to some of the complications of diabetic neuropathy. Hence, we conclude that there should be a regularized screening of serum HCY levels in all diabetes, to prevent complications of diabetic neuropathy.

Hyperhomocysteinemia was an independent risk factor for the occurrence of diabetic neuropathy. Treatment of existing hyperhomocysteinemia with folic acid and Vitamin B12 may be useful in reducing the risk of microvascular complications in T2DM.

\section{ACKNOWLEGMENT}

The authors are very thankful to the Vels Institute of Science Technology and Advanced Studies (VISTAS) and its management for providing research facilities and encouragement.

\section{REFERENCES}

1. Olokoba AB, Obateru OA, Olokoba LB. Type 2 diabetes mellitus: A review of current trends. Oman Med J 2012;27:269-73.

2. Vinik AI, Park TS, Stansberry KB, Pittenger GL. Diabetic neuropathies. Diabetologia 2000;43:957-73.

3. Edwards JL, Vincent AM, Cheng HT, Feldman EL. Diabetic neuropathy: Mechanisms to management. Pharmacol Ther 2008;120:1-34.

4. Callaghan BC, Cheng HT, Stables CL, Smith AL, Feldman EL. Diabetic neuropathy: Clinical manifestations and current treatments. Lancet Neurol 2012;11:521-34.

5. Nygård O, Vollset SE, Refsum H, Stensvold I, Tverdal A, Nordrehaug JE, et al. Total plasma homocysteine and cardiovascular risk profile. The hordaland homocysteine study. JAMA 1995;274:1526-33.

6. Ganguly P, Alam SF. Role of homocysteine in the development of cardiovascular disease. Nutr J 2015;14:6. 
7. Ferri FF, editor. Laboratory Tests and Interpretation of Results. Ferri's Clinical Advisor. $1^{\text {st }}$ ed. Philadelphia, PA: Elsevier Mosby; 2012.

8. Ansari R, Mahta A, Mallack E, Luo JJ. Hyperhomocysteinemia and neurologic disorders: A review. J Clin Neurol 2014;10:281-8.

9. Pirouzpanah S, Taleban FA, Mehdipour P, Atri M, Foroutan-Ghaznavi M. Plasma total homocysteine level in association with folate, pyridoxine, and cobalamin status among iranian primary breast cancer patients. Nutr Cancer 2014;66:1097-108.

10. Brattström L, Lindgren A, Israelsson B, Andersson A, Hultberg B. Homocysteine and cysteine: Determinants of plasma levels in middleaged and elderly subjects. J Intern Med 1994;236:633-41.

11. Arnesen E, Refsum H, Bønaa KH, Ueland PM, Førde $\mathrm{OH}$, Nordrehaug JE, et al. Serum total homocysteine and coronary heart disease. Int J Epidemiol 1995;24:704-9.

12. Christen WG, Ajani UA, Glynn RJ, Hennekens CH. Blood levels of homocysteine and increased risks of cardiovascular disease: Causal or casual? Arch Intern Med 2000;160:422-34.

13. Anand P, Awasthi S, Mahdi A, Tiwari M, Agarwal GG. Serum homocysteine in Indian adolescents. Indian J Pediatr 2009;76:705-9.

14. Januła A, Rujaswini T. Homocysteine and diabetes. Warsaw, Poland: Wiadomosci lekarskie; 1960. p. 58-6.

15. Jacob S. Homocysteine metabolism. Ann Rev Nutr 1999;19:217-46

16. Lentz SR. Mechanisms of homocysteine-induced atherothrombosis. J Thromb Haemost 2005;3:1646-54.

17. Fokkema MR, Gilissen MF, Van Doormaal JJ, Volmer M, Kema IP, Muskiet FA, et al. Fasting vs nonfasting plasma homocysteine concentrations for diagnosis of hyperhomocysteinemia. Clin Chem 2003;49:818-21.

18. Chowdary PR, Praveen D, Aanandhi MV. A prospective study on incidence of dyslipidemia in diabetes mellitus. Res J Pharm Tech 2017;10:431-3.
19. Fotiou P, Raptis A, Apergis G, Dimitriadis G, Vergados I, Theodossiadis P, et al. Vitamin status as a determinant of serum homocysteine concentration in Type 2 diabetic retinopathy. J Diabetes Res 2014;2014:807209.

20. Lakshman R, Negi NS, Gupta B. Prevalence of hyperhomocysteinaemia in Type 2 diabetes mellitus and its correlation with its complications. J Ind Aca Clin Med 2012;13:277-81.

21. Saurabh B, Kapoor SG. Serum homocysteine levels in Type 2 diabetes mellitus patients. 2015;2015:77-83

22. Ambrosch A, Dierkes J, Lobmann R, Kühne W, König W, Luley C, et al. Relation between homocysteinaemia and diabetic neuropathy in patients with Type 2 diabetes mellitus. Diabet Med 2001;18:185-92.

23. Buysschaert $M$, Dramais AS, Wallemacq PE, Hermans MP. Hyperhomocysteinemia in Type 2 diabetes: Relationship to macroangiopathy, nephropathy, and insulin resistance. Diabetes Care 2000;23:1816-22.

24. Heming G, Chen X, Zhang H, Zhang X. Serum homocysteine levels and diabetic neuropathy in patients with Type 2 diabetes mellitus: A systematic review and meta-analysis. Int J Clin Exp Med 2016;9:19588-94.

25. Brazionis L, Rowley K Sr, Itsiopoulos C, Harper CA, O’Dea K. Homocysteine and diabetic retinopathy. Diabetes Care 2008;31:50-6.

26. Alnajjar MK. Homocysteine Levels and Some Biochemical Parameters Among Type 2 Diabetic Nephropathy Patients. Gaza City: Doctoral dissertation, Islamic University-Gaza; 2013.

27. Mundu PA, Kumar B, Mitra JK, Kumar M, Sinha R. Study of Assessment of Plasma Homocysteine Level in Microvascular Complications of Type 2 Diabetes Mellitus; 2017.

28. Hammad RS. Homocysteine and Some Biochemical Parameters in Type 2 Diabetic Patients from Gaza Governorate. Gaza: Islamic University-Gaza. Faculty of Science. Medical Technology; 2013. 